\title{
Femtosecond laser helical drilling of nickel-base single-crystal super-alloy: Effect of machining parameters on geometrical characteristics of micro-holes
}

\author{
Yin, C.P. ${ }^{a}$, Wu, Z.P. ${ }^{a}$, Dong, Y.W. ${ }^{\mathrm{a}, \mathrm{b},{ }^{*}}$, You, Y.C. ${ }^{\mathrm{a}}$, Liao, T. $^{\mathrm{a}}$ \\ ${ }^{a}$ School of Aerospace Engineering, Xiamen University, Xiamen, P.R. China \\ ${ }^{b}$ Shenzhen Research Institute, Xiamen University, Shenzhen, P.R. China
}

\begin{abstract}
A B S T R A C T
Laser micro-hole processing has been widely used in industry. Many laser processing parameters can affect the processing results. The relationship between the geometrical shapes of micro-holes and the laser processing parameters has not been determined accurately. In this paper, experiments on the femtosecond laser drilling of the nickel-base single-crystal super-alloy (DD6) materials were conducted to determine the relationship between the parameters, such as the laser single-pulse energy, rotation rate, and downward focus rate, and the geometrical characteristics of the micro-holes, such as the diameter, and roundness. A group of orthogonal experiments were conducted to determine the effects of the comprehensive influencing factors on the geometrical characteristics of the micro-holes. After the experiments were conducted and analysed, the experimental results were modelled by a backpropagation neural network, and the mapping relationship between the laser parameters and the geometrical morphologies of the micro-holes was constructed. The model established by the backpropagation neural network could obtain accurate prediction results, and the predictions of the diameters of the micro-holes were better than those of the roundness.
\end{abstract}

\section{ARTICLE INFO}

Keywords:

Femtosecond laser;

Micro-hole machining;

Helical drilling;

Nickel-base single-crystal superalloy (DD6);

Orthogonal experiment;

Artificial neural networks (ANN)

*Corresponding author:

yiweidong@xmu.edu.cn

(Dong, Y.W.)

Article history:

Received 26 November 2019

Revised 10 December 2019

Accepted 11 December 2019

\section{References}

[1] Huang, H., Yang, L.-M., Liu, J. (2014). Micro-hole drilling and cutting using femtosecond fiber laser, Optical Engineering, Vol. 53, No. 5, Article No. 051513, doi: 10.1117/1.0E.53.5.051513.

[2] Rihakova, L., Chmelickova, H. (2017). Laser drilling of alumina ceramics using solid state Nd: YAG laser and QCW fiber laser: Effect of process parameters on the hole geometry, Advances in Production Engineering \& Management, Vol. 12, No. 4, 412-420, doi: 10.14743/apem2017.4.268.

[3] Shaegh, S.A.M., Pourmand, A., Nabavinia, M., Avci, H., Tamayol, A., Mostafalu, P., Ghavifekr, H.B., Aghdam, E.N., Dokmeci, M.R., Khademhosseini, A., Zhang, Y.S. (2018). Rapid prototyping of whole-thermoplastic microfluidics with built-in microvalves using laser ablation and thermal fusion bonding, Sensors and Actuators B: Chemical, Vol. 255, Part 1, 100-109, doi: 10.1016/j.snb.2017.07.138.

[4] Padmanabham, G., Bathe, R. (2018). Laser materials processing for industrial applications, Proceedings of the National Academy of Sciences, India Section A: Physical Sciences, Vol. 88, No. 3, 359-374, doi: 10.1007/s40010018-0523-5.

[5] Ciurana, J., Arias, G., Ozel, T. (2009). Neural network modeling and particle swarm optimization (PSO) of process parameters in pulsed laser micromachining of hardened AISI H13 steel, Materials and Manufacturing Processes, Vol. 24, No. 3, 358-368, doi: 10.1080/10426910802679568.

[6] Dubey, A.K., Yadava, V. (2008). Laser beam machining - A review, International Journal of Machine Tools and Manufacture, Vol. 48, No. 6, 609-628, doi: 10.1016/i.ijmachtools.2007.10.017. 
[7] Kamlage, G., Bauer, T., Ostendorf, A., Chichkov, B.N. (2003). Deep drilling of metals by femtosecond laser pulses, Applied Physics A, Vol. 77, No. 2, 307-310, doi: 10.1007/s00339-003-2120-x.

[8] Zoubir, A., Shah, L., Richardson, K., Richardson, M. (2003). Practical uses of femtosecond laser micro-materials processing, Applied Physics A, Vol. 77, No. 2, 311-315, doi: 10.1007/s00339-003-2121-9.

[9] Gruner, A., Schille, J., Loeschner, U. (2016). Experimental study on micro hole drilling using ultrashort pulse laser radiation, Physics Procedia, Vol. 83, 157-166, doi: 10.1016/i.phpro.2016.08.030.

[10] Wang, X.C., Zheng, H.Y., Chu, P.L., Tan, J.L., Teh, K.M., Liu, T., Ang, B.C.Y., Tay, G.H. (2010). Femtosecond laser drilling of alumina ceramic substrates, Applied Physics A, Vol. 101, No. 2, 271-278, doi: 10.1007/s00339-010-5816-8.

[11] Yang, L., Kong, X., Wang, Y., Ding, Y., Zhang, H., Chi, G. (2016). Laser micro-holes machining technology and its application, Aeronautical Manufacturing Technology, No. 19, 271-278, doi: 10.16080/j.issn1671-833x.2016.19. $\underline{032}$.

[12] Liu, Y., Zhang, R., Li, W., Wang, J., Yang, X., Cheng, L., Zhang, L. (2018). Effect of machining parameter on femtosecond laser drilling processing on $\mathrm{SiC} / \mathrm{SiC}$ composites, The International Journal of Advanced Manufacturing Technology, Vol. 96, No. 5-8, 1795-1811, doi: 10.1007/s00170-017-1163-7.

[13] Dausinger, F. (2002). Femtosecond technology for precision manufacturing: Fundamental and technical aspects, In: Proceedings of Third International Symposium on Laser Precision Microfabrication, Osaka, Japan, doi: 10.1117/ 12.486506.

[14] Abeln, T., Radtke, J., Dausinger, F. (1999). High precision drilling with short-pulsed solid-state lasers, In: Laser Institute of America - Proceedings - LIA, Vol. 88, 195-203, doi: 10.2351/1.5059302.

[15] Liao, C., Anderson, W., Antaw, F., Trau, M. (2018). Maskless 3D ablation of precise microhole structures in plastics using femtosecond laser pulses, ACS Applied Materials \& Interfaces, Vol. 10, No. 4, 4315-4323, doi: 10.1021/ acsami.7b18029.

[16] Kraus, M., Ahmed, M.A., Michalowski, A., Voss, A., Weber, R., Graf, T. (2010). Microdrilling in steel using ultrashort pulsed laser beams with radial and azimuthal polarization, Optics Express, Vol. 18, No. 21, 22305-22313, doi: 10.1364/OE.18.022305.

[17] ISO 1101:2017 Geometrical product specifications (GPS) - Geometrical tolerancing - Tolerances of form, orientation, location and run-out, from https://www.iso.org/standard/66777.html, accessed October 27, 2019.

[18] Zhu, J., Chew, D.A.S., Lv, S., Wu, W. (2013). Optimization method for building envelope design to minimize carbon emissions of building operational energy consumption using orthogonal experimental design (OED), Habitat International, Vol. 37, 148-154, doi: 10.1016/j.habitatint.2011.12.006.

[19] Wang, C., Xue, S., Chen, G., Luan, D., Wang, S., Wang, Y., Wang, S., Liu, J., Wang, Z., Zhang, P. (2018). Influence of laser parameters on micro-hole drilling of Cu50Zr50 amorphous alloys foil, Ferroelectrics, Vol. 523, No. 1, 61-66, doi: 10.1080/00150193.2018.1391557.

[20] Fan, N.-N., Xia, Z.-D., Sun, X.-Y., Hu, Y.-W. (2016). Experimental study on stainless steel micro-hole trepanned by femtosecond laser, Laser \& Infrared, Vol. 46, No. 10, 1200-1205, doi: 10.3969/j.issn.1001-5078.2016.10.006.

[21] Fornaroli, C., Holtkamp, J., Gillner, A. (2013). Laser-beam helical drilling of high quality micro holes, Physics Procedia, Vol. 41, 661-669, doi: 10.1016/i.phpro.2013.03.130.

[22] Wang, G.-A., Zhang, Y.-Z., Ni, X.-W., Lu, J. (2007). Effect of deviation distance to focal spot on nanosecond-pulsedlaser drilling rates in air, Chinese Journal of Lasers, Vol. 34, No. 12, 1621-1624.

[23] Zou, Z.-Q., Li, J., Hu, L.-Y. (2017). Diameter changing regularity with the laser parameters of nanosecond laser drilling, Optics \& Optoelectronic Technology, Vol. 15, No. 5, 58-61.

[24] Verbeeck, J., Bertoni, G., Schattschneider, P. (2008). The Fresnel effect of a defocused biprism on the fringes in inelastic holography, Ultramicroscopy, Vol. 108, No. 3, 263-269, doi: 10.1016/i.ultramic.2007.06.007.

[25] Wu, X., Leung, D.Y.C. (2011). Optimization of biodiesel production from camelina oil using orthogonal experiment, Applied Energy, Vol. 88, No. 11,3615-3624, doi: 10.1016/j.apenergy.2011.04.041.

[26] Li, X.J., Dong, Y.W., Yin, C.P., Zhao, Q., You, Y.C. (2018). Geometric parameters evolution experiment of hole during femtosecond laser helical drilling, Chinese Journal of Lasers, Vol. 45, No. 5, doi: 10.3788/CJL201845.0502008.

[27] Ren, N., Zhang, L., Wang, H., Xia, K., Shi, C. (2017). Orthogonal experiments and variance analysis in Nd:YAG pulsed laser trepanning drilling, Laser \& Optoelectronics Progress, Vol. 54, No. 6, doi: 10.3788/LOP54.061408.

[28] Dhara, S.K., Kuar, A.S., Mitra, S. (2008). An artificial neural network approach on parametric optimization of laser micro-machining of die-steel, The International Journal of Advanced Manufacturing Technology, Vol. 39, No. 1-2, 39-46, doi: 10.1007/s00170-007-1199-1.

[29] Casalino, G. (2018). [INVITED] Computational intelligence for smart laser materials processing, Optics \& Laser Technology, Vol. 100, 165-175, doi: 10.1016/i.optlastec.2017.10.011.

[30] Majumder, A. (2010). Comparison of ANN with RSM in predicting surface roughness with respect to process parameters in Nd:YAG laser drilling, International Journal of Engineering Science and Technology, Vol. 2, 51755186.

[31] Li, M., Wu, H., Wang, Y., Handroos, H., Carbone, G. (2017). Modified Levenberg-Marquardt algorithm for backpropagation neural network training in dynamic model identification of mechanical systems, Journal of Dynamic Systems, Measurement, and Control, Vol. 139, No. 3, Article No. 031012, doi: 10.1115/1.4035010.

[32] Guo, Q.-C., He, Z.-F. (2014). Economic forecasting model based on artificial neural network, Computing Technology and Automation, Vol. 33, No. 1, 132-136.

[33] Zhang, Y., Gao, X., Katayama, S. (2015). Weld appearance prediction with BP neural network improved by genetic algorithm during disk laser welding, Journal of Manufacturing Systems, Vol. 34, 53-59, doi: 10.1016/i.jmsy.2014. $\underline{10.005}$.

[34] Ding, S., Su, C., Yu, J. (2011). An optimizing BP neural network algorithm based on genetic algorithm, Artificial Intelligence Review, Vol. 36, No. 2, 153-162, doi: 10.1007/s10462-011-9208-z. 
[35] Ai, J.L., Yang, X.Z. (2017). Fault diagnosis of aero-engine based on self-adaptive neural network, Scientia Sinica Technologica, Vol. 48, No. 3, 326-335, doi: 10.1360/N092017-00224. 


\title{
Spiralno vrtanje monokristalne superzlitine na osnovi niklja s femtosekundnim laserjem: Vpliv obdelovalnih parametrov na geometrijske značilnosti mikrolukenj
}

\author{
Yin, C.P. ${ }^{a}$, Wu, Z.P. ${ }^{a}$, Dong, Y.W. ${ }^{\mathrm{a}, \mathrm{b},{ }^{*},}$, You, Y.C. ${ }^{a}$, Liao, T. ${ }^{a}$ \\ ${ }^{a}$ School of Aerospace Engineering, Xiamen University, Xiamen, P.R. China \\ ${ }^{b}$ Shenzhen Research Institute, Xiamen University, Shenzhen, P.R. China
}

\section{POVZETEK}

Laserska izdelava mikrolukenj se v industriji pogosto uporablja. Številni parametri laserske obdelave lahko vplivajo na rezultate obdelave. Razmerje med geometrijskimi oblikami mikrolukenj in parametri laserske obdelave niso natančno določeni. $V$ tem prispevku so bili izvedeni poskusi na femtosekundnem laserskem vrtanju monokristalne superzlitine niklja (DD6) za določitev razmerja med parametri, kot so impulzna energija laserja, hitrost vrtenja in stopnja globinske osredotočenosti ter geometrijskimi značilnostmi mikrolukenj, kot sta premer in krožnost. Izvedena je bila skupina pravokotnih eksperimentov za določitev vplivov parametrov na geometrijske značilnosti mikrolukenj. Po izvedbi in analizi eksperimentov smo eksperimentalne rezultate modelirali z nevronsko mrežo z vzvratnim razširjanjem in konstruirali povezavo med laserskimi parametri in geometrijskimi morfologijami mikrolukenj. Model, ki ga je vzpostavila nevronska mreža, je natančno napovedal rezultate, napovedi premerov mikrolukenj pa so bile boljše od napovedi krožnosti.

(C) 2019 CPE, University of Maribor. All rights reserved.

\section{PODATKI O ČLANKU}

Ključne besede:

Femtosekundni laser;

Obdelava mikrolukenj;

Spiralno vrtanje;

Monokristalna super-zlitina na osnovi niklja (DD6);

Pravokotni eksperiment; Umetne nevronske mreže (ANN)

*Kontaktna oseba:

yiweidong@xmu.edu.cn (Dong, Y.W.)

\section{Zgodovina članka:}

Prejet 26. novembra 2019

Popravljen 10. decembra 2019

Sprejet 11. decembra 2019 